\title{
Building Microclouds at the Network Edge with the Cloudy Platform
}

\author{
Felix Freitag, Roger Pueyo, Leandro Navarro \\ Department of Computer Architecture. Technical University of Catalunya. Barcelona, Spain \\ \{felix, rpueyo, leandro\}@ac.upc.edu
}

\begin{abstract}
Edge computing enables new types of services which operate at the network edge. There are important use cases in pervasive computing, ambient intelligence and the Internet of Things (IoT) for edge computing. In this demo paper we present microclouds deployed at the networks edge in the Guifi.net community network leveraging an open extensible platform called Cloudy. The demonstration focuses on the following aspects: The usage of Cloudy for end users, the services of Cloudy to build microclouds, and the application scenarios of IoT data management within microclouds.

Index Terms - edge computing; community clouds;
\end{abstract}

\section{INTRODUCTION}

Important use cases in the Internet of Things (IoT) with huge business potential drive edge computing research and deployment. Societal needs to achieve through smart cities and smart homes more sustainable solutions supported by distributed ICT infrastructures. Edge computing is already applied in many industrial and consumer-oriented scenarios.

In edge computing an additional infrastructure layer is added to the cloud infrastructure by by placing computing devices at the network edge. On these edge devices, specific functions are performed as part of a cloud service leveraging their closeness to the users or the data source.

Vendor solutions provide edge computing platforms to solve specific requirements. In order to satisfy new user requirements, the possibility of extending edge devices with additional services, however, should also be provided. For instance, in the scenario of smart homes, it must be possible to add evolving privacy and security needs to a given service solution, which could be enabled through an open edge computing platform.

Edge devices, however, have limited resource capabilities. While data center resources do provide elasticity, an edge devices cannot scale to workloads which exceed its capacity. One solution to this situation is trying to split larger task and outsource non-critical parts to data centers [2]. Another approach, which we present here, is to improve edge device elasticity by microclouds, which pool edge resources and, through an open software platform, allow service providers and end users to run edge-centric services.

In this demo paper, we present edge microclouds built with the Cloudy platform ${ }^{1}$. Our approach brings together the concepts of community cloud computing [1] and edge computing

${ }^{1}$ http://cloudy.community/
[3], materialized as microclouds which are deployed in the Guifi.net community network ${ }^{2}$.

\section{Microclouds AT THE NETWORK EDGE}

\section{A. Microclouds in Guifinet}

Figure 1 illustrates the concept of microclouds. Different hardware devices are used as nodes to build the microclouds. These microclouds consist of interconnected edge nodes which allow to share resources and services among its users. Different to Fog computing [5], where the Fog device establishes a vertical interconnection with data center services, in the microclouds the cloud is formed horizontally among edge devices. The cloud nodes are spread over the edge network at different geographic locations. An important feature of the microcloud infrastructure is that it is not bound to a certain number of devices, but grows organically by every new node that is added to the microcloud infrastructure.

We demonstrate microclouds by an deployment in the Guifi.net community network. Community networks consist of IP-based communication networks which local communities of citizens build and operate by themselves. Often they providing Internet access to the population of areas which are unattended by commercial telecom operators. Guifi.net is the community network where we deployed the microcloud infrastructure. With more than 30000 nodes, Guifi.net can be considered the largest community network worldwide. Devices which are part of the microclouds consist mostly of mini-PCs, which are located at end user premises, but also of some desktop PCs, which are mainly in municipality buildings.

\section{B. The Cloudy distribution}

The software, which we have developed to build microclouds is a Debian-based Linux platform called Cloudy. Cloudy can be obtained from public repositories ${ }^{3}$. The Cloudy distribution is installed on each of the devices connected to the microcloud.

Service extensions are possible. While there is a preconfigured set of services integrated in the Cloudy distribution, the microcloud formed by the Cloudy platform is open to provide new services contributed by its users. The Guifi community cloud currently runs on around thirty devices.

\footnotetext{
${ }^{2}$ http://guifi.net/

${ }^{3}$ https://github.com/Clommunity/
} 


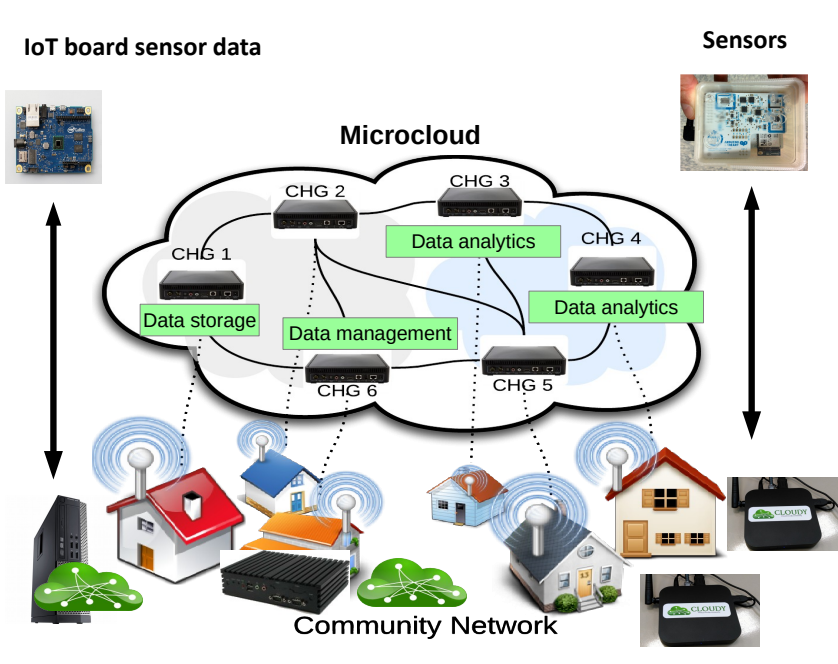

Fig. 1. Edge microcloud on heterogeneous hardware for IoT applications.

Instantaneous information on services and devices can be obtained at any moment from a publicly available Cloudy instance.

\section{IOT SCENARIO FOR MICROCLOUDS}

\section{A. Service provision with Docker containers}

For Cloudy we have chosen Docker as technology to support users with the means to achieve personalization and customized service deployments. The Cloudy user can find a list of pre-configured applications available that can be started in Docker containers. These applications can be installed and un-installed with a click on the Web interface. The user can also choose if these applications should be published, and therefore potentially shared, with the users of the microcloud.

\section{B. Cloudy usage}

Services in Cloudy are configured through its GUI. The integration of Docker in the Cloudy GUI targets at achieving an end user-friendly Docker configuration and control. To this end, the approach we followed is to hide the technical complexity of the Docker usage behind graphical Web interfaces, more suitable for end users. Therefore, flexible service provision is achieved by leveraging Docker containers, which the integrating in Cloudy has made accessible to be used by end-users [1].

Figure 2) shows a screenshot, where five main types of services can be observed. From top left side of Figure 2) these are: 1) Search, 2) Guifi.net, 3) Personal cloud, 4) Community cloud and 5) Enterprise cloud. Specifically in the Enterprise cloud, applications available as Docker images can be launched.

Figure 2 shows the MQTT Mosquitto broker deployed as Docker container and available as service in the microcloud. While this broker is available for IoT data publishers and subscribers, it is interesting to note that this service is made available within the microcloud to support users with IoT data management.

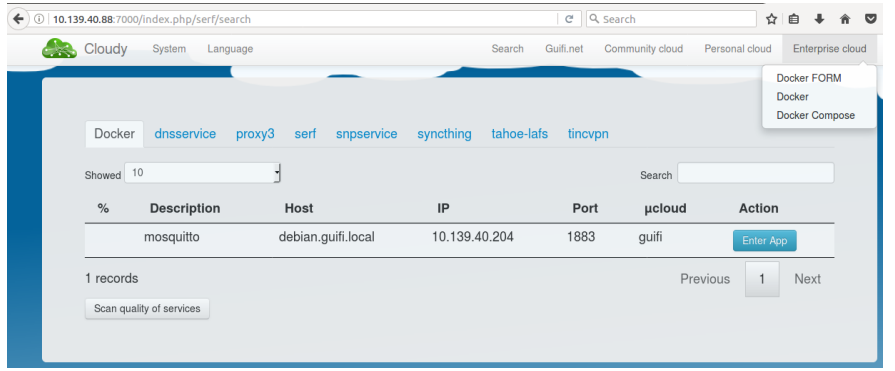

Fig. 2. The Search service finds the Mosquitto broker deployed as Docker container.

Figure 1 illustrates other components with which a local microcloud could support IoT data management. While specific platform such as a storage service implemented through TahoeLAFS is already available in Cloudy, other functions such as machine learning may become available for the network edge in the near future as to current research in deep learning [4] .

\section{EXPERIMENTATION}

The experimentation with the presented microclouds can be done from the following perspectives:

1) Live access to the microcloud in Guifi.net, as seen by the community network cloud user who hosts a Cloudy node ${ }^{4}$. By connecting to the Web-GUI of Cloudy instances, the cloud instances and services deployed in the Guifi.net microcloud can be explored.

2) Installation to build a Cloudy node and experimentation with the services to support microclouds.

3) Service extensions to support specific scenarios such as IoT.

\section{ACKNOWLEDGMENT}

This work was partially supported by the European Union's Horizon 2020 research and innovation programme under the project netCommons (H2020-688768), the project FIESTA (H2020-643943), and by the Spanish government under contract TIN2016-77836-C2-2-R.

\section{REFERENCES}

[1] R. Baig, F. Freitag, and L. Navarro, "Fostering collaborative edge service provision in community clouds with docker," in 2016 IEEE International Conference on Computer and Information Technology (CIT), Dec 2016, pp. 560-567.

[2] N. Powers and T. Soyata, "Axaas (acceleration as a service): Can the telecom service provider rent a cloudlet?" in 2015 IEEE 4th International Conference on Cloud Networking (CloudNet), Oct 2015, pp. 232-238.

[3] W. Shi and S. Dustdar, "The promise of edge computing," Computer, vol. 49, no. 5, pp. 78-81, May 2016.

[4] S. Teerapittayanon, B. McDanel, and H. T. Kung, "Distributed deep neural networks over the cloud, the edge and end devices," in 2017 IEEE 37th International Conference on Distributed Computing Systems (ICDCS), June 2017, pp. 328-339.

[5] B. Varghese, N. Wang, D. S. Nikolopoulos, and R. Buyya, "Feasibility of fog computing," CoRR, vol. abs/1701.05451, 2017. [Online]. Available: http://arxiv.org/abs/1701.05451

\footnotetext{
${ }^{4}$ Access is available through a publicly available Cloudy instance at http: //demo.cloudy.clommunity/, with login guest:guest.
} 